\title{
Mechanism of inhibiting proliferation of hepatocellular carcinoma Hepa1-6 cells by embryonic stem cell-conditioned medium
}

\author{
LONGQIN LI, YICHAO ZHENG, QI ZHENG and JIAJI JIANG \\ Liver Research Center, First Affiliated Hospital of Fujian Medical University, Fuzhou, Fujian 350005, P.R. China
}

Received December 5, 2018; Accepted August 23, 2019

DOI: $10.3892 /$ etm.2020.8527

\begin{abstract}
The present study aimed to investigate the antiproliferative effect of embryonic stem cell-conditioned medium (ESC-CM) on the mouse liver cancer Hepa1-6 cells in vitro. Furthermore, in order to elucidate the underlying molecular mechanism, the microRNAs (miRNAs) in ESC-CM associated with the inhibition of Hepa1-6 proliferation were identified. Following the co-culture of ESC-CM and Hepa1-6 in Transwell chambers, the proliferation, cell cycle, apoptosis and associated protein expression were determined in Hepal- 6 cells Moreover, miRNA array analysis was employed to identify differentially expressed miRNAs. Based on the differentially expressed miRNAs, the target genes and potential associated signaling pathways were determined. Finally, RT-qPCR was conducted to confirm the above results. The ESC-CM inhibited Hepal- 6 cell proliferation and increased the percentage of cells at $G_{1}$ phase and decreased the percentage of cells at the $\mathrm{G}_{2} / \mathrm{M}$ phase of the cell cycle. The expression of cyclin D1/cyclin-dependent kinase (CDK)4/CDK6 was decreased following co-culture, with no effect on cell apoptosis. Six significantly regulated miRNAs were identified and 423 putative target genes of these regulated miRNAs were predicted. Gene ontology analysis revealed the putative target genes to be associated with the 'DNA replication (GO: 0006260)' GO term, 'apoptosis' and 'signal transduction'. The Kyoto Encyclopedia of Genes and Genomes analysis indicated that deregulated miRNAs were enriched in the Wnt signaling (KEGG entry: Map 04310) and Hippo signaling pathways (KEGG entry: Map 04390), pathways associated with cancer. Overall, the present study demonstrated the inhibition of Hepa1- 6 cell line proliferation upon treatment with ESC-CM, by decreasing cell cycle-associated protein cyclin D1/CDK4/CDK6 expression and arresting cells in $\mathrm{G}_{1}$ phase of the cell cycle, with no effect on cell apoptosis. Furthermore, the inhibition of proliferation
\end{abstract}

Correspondence to: Dr Jiaji Jiang, Liver Research Center, First Affiliated Hospital of Fujian Medical University, 20 Chazhong Road, Fuzhou, Fujian 350005, P.R. China

E-mail: jiangjiaji2018@yeah.net

Key words: embryonic stem cell conditioned medium, liver cancer, cell cycle, proliferation, microRNAs by ESC-CM may be mediated by miRNAs that affect cell cycle-associated mRNAs and the Wnt signaling pathway.

\section{Introduction}

Primary liver cancer is a common malignant tumor, ranking sixth in the global incidence of cancer and fourth in tumor mortality in 2018 (1). Liver cancer is characterized by high rates of recurrence and high metastasis, severely hindering the overall efficacy of the treatment methods for this disease (2).

Embryonic stem cells (ESCs) are derived from the inner cell mass of blastocyst and have self-renewal, unlimited proliferation and potential differentiation abilities (3). Some types of tumor cells can form teratomas in vivo that are insensitive to growth signal inhibition and cell death stimuli (4), suggesting that cancer and ESCs share similar phenotypes in terms of cell differentiation, proliferation and cell invasion. In addition, normal tadpoles could be obtained by transplanting the nucleus of a Lucke's kidney cancer cell into a denuclearized fertilized egg, where no tumor tissue was formed (3). A study by Illmensee (5) demonstrated that other embryonic tissue cells, except for embryonic cells, do not possess this ability, and indicated that tumor cells can also be induced to differentiate into mature histiocytes under specific conditions.

The tumor microenvironment, consisting of microvasculature, extracellular matrix and various stromal cells (tumor-associated fibroblasts, mesenchymal stem cells and endothelial cells) and signaling molecules secreted by these cells, play an important role in the process of tumorigenesis, development and metastasis $(5,6)$. ESC conditioned medium (ESC-CM) could be used to simulate the ESC microenvironment in vitro (7).

Giuffrida et al (7) revealed that ESC-CM can inhibit the proliferation of ovarian cancer cells by regulating the cell cycle, which was associated with the secretion of small molecules by ESCs. The ability of ESC-CM to inhibit the proliferation and invasion of tumor cells is associated with the secretion of lefty A by ESCs (8). The proliferation of breast cancer is also inhibited in ESC-CM (9). ESC-CM resulted in decreased cancer cell migration, invasion, angiogenesis and decreased the ability of tumor formation following subcutaneous transplantation in mice. The antitumor effects of ESC-CM were mediated by inhibition of tumor cell proliferation, angiogenesis, migration, and STAT3 signaling pathway (8). 
Exosomes serve important roles in extracellular signal transduction in both tumor and normal cells (10), which includes a number of bioactive substances such as heat shock proteins and microRNAs (miRNAs) (11). miRNAs are endogenous small RNAs $20-24$ nucleotides in length and have important regulatory functions in the cell. miRNAs are formed by multi-step digestion in cells, which involves the formation of pri-miRNA, pre-miRNA and finally mature miRNA. miRNA 290-295 in the exosomes derived from ESCs, particularly miRNA 294, have been shown to ameliorate myocardial infarction in mice (12). miRNA 294 was demonstrated to improve myocardial angiogenesis and myocardial cell viability, and decrease myocardial fibrosis, following myocardial infarction.

The inoculation of animals with ESCs can effectively prevent the occurrence of colon (9), lung (10) and ovarian cancer (11). ESCs have therapeutic effects on early tumors with low tumor burden and can effectively decrease the incidence of inflammation-associated tumors (13); however, the underlying mechanisms are unknown.

To date, the regulation of tumor cell miRNAs by ESC-CM has been poorly investigated (12). In the present study, ESCs and hepatocellular carcinoma Hepal-6 cells were co-cultured via non-direct contact, in order to investigate the inhibitory effect of ESC-CM on the biological behavior of liver tumor cells in vitro. By comparing the tumor cell miRNA expression profile between ESC-CM treatment and mouse embryonic fibroblast (MEF)-CM treatment, the possible miRNAs underlying the regulatory mechanisms were explored. The findings of the present study can help determine the association between miRNAs and the malignant behaviors of tumors.

\section{Materials and methods}

Materials. MTT was obtained from Sigma-Aldrich (Merck KGaA) and Transwell chambers with $0.4-\mu$ m pore sizes were purchased from Corning Inc. Cell cycle and apoptosis analysis (cat. no. C1052) and Annexin V-Phycoerythrin Apoptosis Detection Kits (cat. no. C1065L) were purchased from Beyotime Institute of Biotechnology. Antibodies against $\beta$-actin, cyclin-dependent kinase (CDK)2, CDK4, CDK6, cyclin D1 and cyclin E1 were purchased from Cell Signaling Technology, Inc.

Cell lines and culture conditions. ESCs and MEFs were supplied by Cyagen Biosciences, Inc. MEFs were cultured in the media of mouse embryonic fibroblast basal medium, $10 \%$ FBS, $1 \%$ glutamine and $100 \mathrm{U} / \mathrm{ml}$ penincillin-streptomycin. The C57BL/6 ESCs were cultured on plates pre-coated with gelatin solution, irradiated C57BL/6 MEFs as feeder cells and mouse ESCs medium (mESC basal medium, $15 \%$ fetal bovine serum, penincillin-streptomycin, $1 \%$ glutamine, nonessential amino acid, 1,000 U/ml leukemia inhibitory factor, $0.1 \mathrm{mM}$ 2-mercaptoethanol; all medium obtained from Cyagen Bioscience Inc.). Hepa1-6 cells were purchased from the Cell Bank of Type Culture Collection of the Chinese Academy of Sciences, maintained in Dulbecco's modified Eagle's medium (DMEM) with high glucose supplemented with $10 \%$ heat-inactivated FBS (both obtained from Gibco; Thermo Fisher Scientific, Inc.) at $37^{\circ} \mathrm{C}$ in a humidified atmosphere containing $5 \% \mathrm{CO}_{2}$.
CM culture. ESC-CM was obtained by overlaying MEF cells with ESCs in the aforementioned mouse ESC growth medium. for 24,48 or $72 \mathrm{~h}$ (days 1, 2 and 3 ESC-CM). Control CM was made by incubating MEFs with stem cell medium for 2448 or $72 \mathrm{~h}$ (day 1, 2 and 3 MEF-CM). Feeder and ESCs were cultured in $90-\mathrm{mm}$ plates containing $10 \mathrm{ml}$ stem cell medium. Feeders were plated at $8 \times 10^{5}$ cells per plate and ESCs were plated at $2 \times 10^{5}$ cells per plate. CM was harvested and passed through a syringe filter to remove any cellular debris.

Hepal-6 and ESCs co-culture. The 24-well Transwell chambers (pore size, $0.4 \mu \mathrm{m}$; membrane diameter, $6.5 \mathrm{~mm}$ ) were purchased from Corning, Inc. When the pore size of the co-culture system was $<3.0 \mu \mathrm{m}$, the cells could not pass through. In this co-culture system, ESCs were seeded into the lower chamber whilst the Hepal- 6 cells were seeded into the upper chamber. Hepa1-6 cells were plated in $2 \mathrm{ml}$ DMEM medium supplemented with $10 \%$ heat-inactivated FBS at a density of $5 \times 10^{5}$ cells/well in the chamber. ESCs on feeder cells and feeder cells only were separately plated at a density of $1 \times 10^{5}$ cells in culture plate, where was under chamber. These cells were allowed to attach overnight. Following $24 \mathrm{~h}$ incubation, DMEM was replaced by stem cell medium and chambers with feeders only or ESCs on feeders were placed in wells containing cancer cells. Co-culture occurred for 24-144 h wherein medium was changed every $24 \mathrm{~h}$. The cell number was assessed at 24, 48, 72, 96, 120 and $144 \mathrm{~h}$ using the MTT method.

Cell proliferation assay. The effect of ESC-CM on Hepa1-6 cell proliferation was measured by MTT assay. Cells were plated in 96-well plates at a density of 2,500 cells/well overnight, following which they were treated with day 1,2 and 3 ESC-CM and MEF-CM. Following incubation for 24, 48, $72,96,120$ or $144 \mathrm{~h}$ at $37^{\circ} \mathrm{C}$ in a humidified incubator, MTT ( $5 \mathrm{mg} / \mathrm{ml}$ in PBS) was added to each well and incubated for $4 \mathrm{~h}$. Subsequently, the medium was removed and $0.1 \mathrm{ml}$ of buffered DMSO was added per well. The absorbance was recorded on a microplate reader (Sepctra Max M2e; Molecular Devices, LLC) at the wavelength of $490 \mathrm{~nm}$. The proliferation curve was generated with time on the horizontal axis and OD value on the vertical axis.

Cell cycle analysis. Following treatment with CM, the DNA content and cell cycle distribution of Hepa1-6 cells were determined by flow cytometry. Cells plated at a density of $5 \times 10^{5}$ cells/well in six-well plates were treated with ESC-CM or MEF-CM for $48 \mathrm{~h}$ and harvested at 24, 48, 72 or $96 \mathrm{~h}$. The cells were washed in PBS and then fixed in cold $70 \%$ ethanol and stored at $4^{\circ} \mathrm{C}$ for $30 \mathrm{~min}$. The cells were washed with cold PBS and resuspended in PBS solution containing $50 \mu \mathrm{g} / \mathrm{ml}$ phycoerythrin (PE) and $100 \mathrm{U} / \mathrm{ml}$ of RNase type A. Cells were then incubated in the dark for $30 \mathrm{~min}$ at $37^{\circ} \mathrm{C}$. Cell cycle was analyzed by flow cytometry (BD Biosciences) and the FlowJo 7.6.1 software (version 7.6.1; FlowJo LLC).

Quantification of apoptosis. For apoptosis analysis, $5 \times 10^{5}$ Hepa1-6 cells/well were plated on six-well plates and treated with ESC-CM or MEF-CM for $48 \mathrm{~h}$ and harvested at 24, 48, 72 or $96 \mathrm{~h}$. The cells were labeled with annexin V and PE. Apoptosis rates were determined by flow cytometry (BD 
Biosciences) and analyzed using the FlowJo software. The percentage of early apoptosis was calculated by counting annexin V-positive and PI-negative cells, and the percentage of the late apoptosis was calculated using annexin V-positive and PI-positive cells.

Western blotting. Hepa1-6 cells treated with MEF-CM or ESC-CM for $72 \mathrm{~h}$ were lysed in RIPA buffer $(150 \mathrm{mM} \mathrm{NaCl}$, $0.5 \%$ sodium deoxycholate, $0.1 \%$ SDS, $1 \%$ NP40, 1 mM EDTA and $50 \mathrm{mM}$ Tris $\mathrm{pH} 8.0$ ). The protein content was determined using the Bicinchoninic Acid protein assay kit (Beyotime Institute of Biotechnology). Equivalent amounts of protein $(50 \mu \mathrm{g})$ were separated by $10 \%$ SDS-PAGE gel and transferred to polyvinylidenedifluoride (PVDF) membranes. The membranes were incubated with blocking buffer (5\% nonfat dry milk) for $2 \mathrm{~h}$ at $4^{\circ} \mathrm{C}$ and then incubated with primary antibodies against Cyclin D1 (1:1,000; cat. no. 26939-1-AP; Proteintech Group, Inc.), Cyclin E1 (1:1,000; cat. no. 11554-1-AP; Proteintech Group, Inc.), CDK2 (1:1,000; cat. no. 2546T; Cell Signaling Technology, Inc.), CDK4 (1:1,000; cat. no. 12790T; Cell Signaling Technology, Inc.), CDK6 (1:1,000; cat. no. 13331T; Cell Signaling Technology, Inc.) or $\beta$-actin (1:5,000; cat. no. A5441; Sigma Aldrich; Merck KGaA) overnight at $4^{\circ} \mathrm{C}$. The membranes were then incubated with horseradish peroxidase-conjugated anti-mouse (1:5,000; cat. no. HAF007, R\&D systems, Inc.) or anti-rabbit (1:5,000; cat. no. HAF008; $\mathrm{R} \& \mathrm{D}$ systems, Inc.) IgG for $1 \mathrm{~h}$ at $37^{\circ} \mathrm{C}$. The blots were subsequently detected using chemiluminescence (BeyoECL plus kit, Beyotime Institute of Biotechnology) and analyzed by Image $\mathrm{J}$ version 18.0 (National Institutes of Health).

Reverse transcription-quantitative- $P C R$ (RT-qPCR). Total RNA was obtained from cancer cells cultured with ESC-CM for $48 \mathrm{~h}$ or MEF-CM for $72 \mathrm{~h}$ using TRIzol ${ }^{\circledR}$ reagent (Invitrogen; Thermo Fisher Scientific, Inc.) or miRNeasy mini kit (Qiagen $\mathrm{GmbH}$ ) according to corresponding manufacturers' protocols. Quantification was performed using a two-step reaction process: Reverse transcription (RT) and PCR. Each RT reaction consisted of $1 \mu \mathrm{g}$ RNA, $4 \mu \mathrm{l}$ miScript HiSpec Buffer, $2 \mu \mathrm{l}$ nucleic acid Mix and $2 \mu \mathrm{l}$ miScript Reverse Transcriptase Mix (Qiagen $\mathrm{GmbH}$ ), in a total volume of $20 \mu \mathrm{l}$ per reaction. Reactions were performed in a GeneAmp ${ }^{\circledR}$ PCR System 9700 (Applied Biosystems; Thermo Fisher Scientific, Inc.) for $60 \mathrm{~min}$ at $37^{\circ} \mathrm{C}$, followed by heat inactivation of RT for $5 \mathrm{~min}$ at $95^{\circ} \mathrm{C}$. The $20 \mu \mathrm{l}$ RT reaction mix was then diluted $5 \mathrm{X}$ in nuclease-free water and held at $-20^{\circ} \mathrm{C}$. Subsequent qPCR was performed using LightCycler ${ }^{\circledR} 480$ II Real-time PCR Instrument (Roche Diagnostics) with $10 \mu \mathrm{l}$ PCR reaction mixture that included $1 \mu \mathrm{l}$ cDNA, $5 \mu 1$ 2X LightCycler ${ }^{\circledR} 480$ SYBR Green I Master (Roche Diagnostics), $0.2 \mu \mathrm{l}$ universal primers (Qiagen $\mathrm{GmbH}$ ), $0.2 \mu \mathrm{l}$ microRNA-specific primers and $3.6 \mu$ nuclease-free water. The thermocycling conditions were as follows: Initial denaturation at $95^{\circ} \mathrm{C}$ for $10 \mathrm{~min}$, followed by 40 cycles of $95^{\circ} \mathrm{C}$ for $10 \mathrm{sec}$ and $60^{\circ} \mathrm{C}$ for $30 \mathrm{sec}$. Each sample was run in triplicate. At the end of the PCR cycles, melting curve analysis was performed to validate the specific generation of the expected PCR product. The microRNA-specific primer sequences were designed in the laboratory and synthesized by Generay Biotech Co., Ltd. based on the microRNA sequences obtained from the miRBase database (ftp://mirbase.org/pub/mirbase/20/), which were listed

Table I. Primer sequences used for reverse transcriptionquantitative PCR.

Primer name $\quad$ Primer sequence (5'-3')

U6

Mmu-miR-10a-5p

Mmu-miR-1187

Mmu-miR-134-5p

Mmu-miR-29b-1-5p

Mmu-miR-3070b-3p

Mmu-miR-421-3p

Mmu, mus musculus; miR, microRNA.

in Table I. The expression levels of microRNAs were normalized to U6) and were calculated using the $2^{-\Delta \Delta \mathrm{Cq}}$ method (14).

miRNA experiment and data analysis. Total RNA was extracted with TRIzol ${ }^{\circledR}$ (Invitrogen; Thermo Fisher Scientific, Inc.) and miRNeasy mini kit (Qiagen $\mathrm{GmbH}$ ). RNA levels were quantified by the NanoDrop ${ }^{\text {TM }} 2000$ (Thermo Fisher Scientific, Inc.) and the RNA integrity was assessed using Agilent Bioanalyzer 2100 (Agilent Technologies, Inc.). The sample labeling, microarray hybridization and washing were performed based on the manufacturer's standard protocols. Briefly, total RNA was dephosphorylated, denatured and then labeled with Cyanine-3-CTP using Low Input QuickAmp Labeling Kit, one-Color (cat. no. 5190-2305; Agilent technologies, Inc.). Following purification, the labeled RNAs were hybridized onto SurePrint Mouse microRNA microarrays (cat. no. G4872A-046065; Agilent technologies, Inc.). After washing, the arrays were scanned with the Agilent Scanner G2505C (Agilent Technologies, Inc.). The associated differentially expressed miRNA primer sequences that were used for qPCR are provided in Table I.

The Feature Extraction software (version 10.7.1.1; Agilent Technologies, Inc.) was used to analyze array images to obtain raw data. The Genespring software (version 12.5; Agilent Technologies, Inc.) was used to complete the basic analysis with the raw data. Initially, the raw data were normalized with the quantile algorithm. The probes with at least $100 \%$ of samples in any one condition out of two conditions and flagged as 'detected' were chosen for further data analysis. Differentially expressed miRNAs were subsequently identified through fold change as well as P-value calculated using t-test. The threshold set for upregulated and downregulated genes was a fold change $>2.0$ and a $\mathrm{P}<0.05$. The target genes of differentially expressed miRNAs were the intersection of miRNAs predicted by three databases (Targetscan version 6.0; http://www.targetscan.org; microRNAorg version 6.2, http://www.microrna.org; Pita release 2010, https://omictools.com/pita-tool). Gene Ontology (GO, release number 2019-07-01; http://geneontology.org/) and Kyoto Encyclopedia of Genes and Genomes (KEGG, release number 2019-10-01; https://www.kegg.jp/) analysis were applied to determine the roles of these target genes. Hierarchical clustering was performed to show the distinguishable miRNA expression pattern among samples. 
A

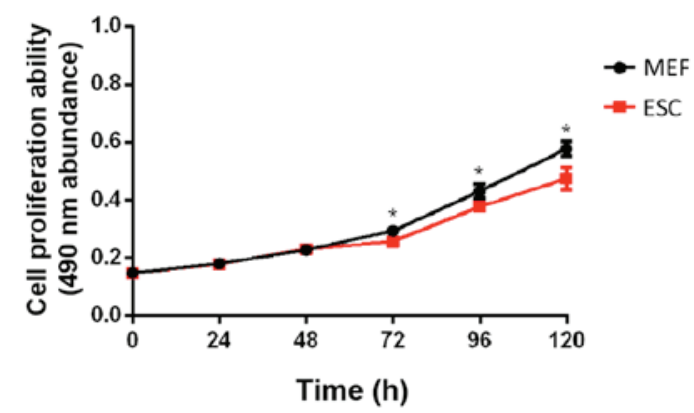

C

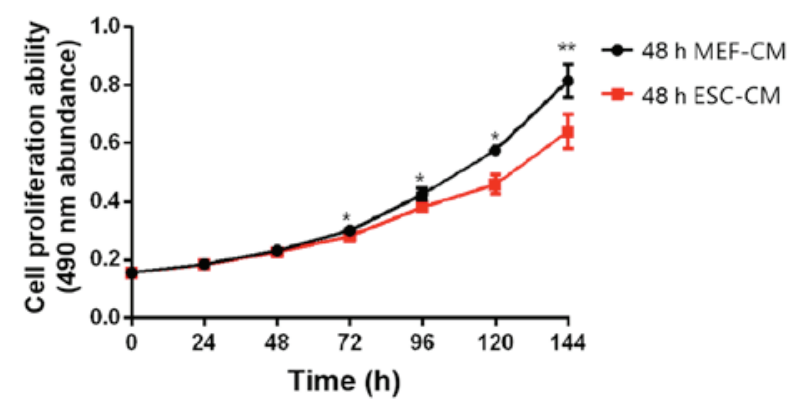

B

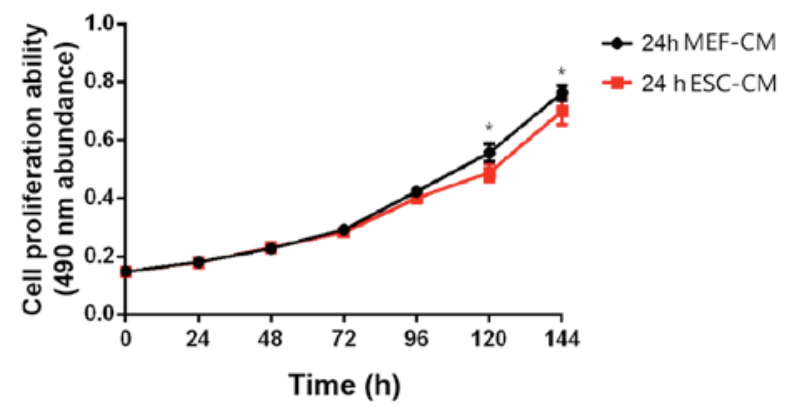

D

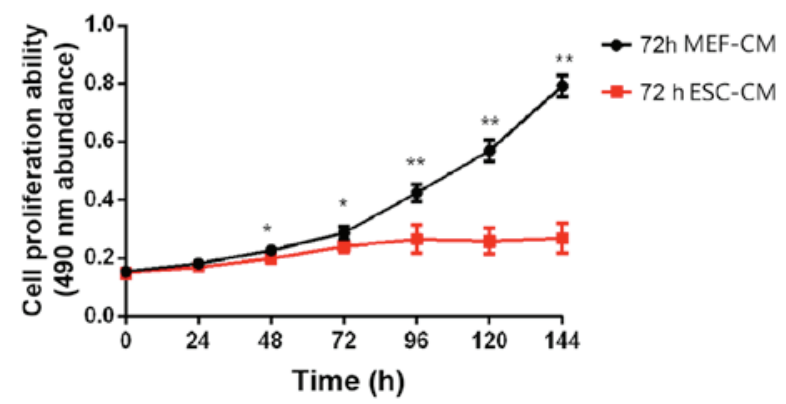

Figure 1. ESC-CM inhibits Hepa1-6 cell proliferation. (A) Hepa1-6 cells co-cultured with ESC or MEFs, respectively. (B) Hepa1-6 cells cultured with 24-h, (C) 48-h and (D) 72-h ESC-CM through a Transwell chamber. Data shown are representatives of three experiments. ${ }^{*}<<0.05$ and ${ }^{* *} \mathrm{P}<0.01$ vs. the respective ESC group. ESC-CM, embryonic stem cell conditioned medium; MEF, mouse embryonic fibroblast.

Statistical analysis. Data are presented as the mean \pm standard deviation. SPSS 16.0 (SPSS, Inc.) and GraphPad Prism 6.0 (GraphPad Software, Inc.) were used for the statistical analysis and graphical display of data, respectively. Each experiment was repeated three times. The differences between groups were analyzed by Student's t-test. $\mathrm{P}<0.05$ was considered to indicate a statistically significant difference.

\section{Results}

ESCs secrete factors that inhibit Hepal-6 proliferation. To determine whether mouse ESCs secreted factors that inhibit Hepa1-6 proliferation, Transwell chambers were used to perform co-culture experiments. The chambers separated ESCs from Hepa1-6 cells by a $0.4-\mu$ m pore, high-density membrane, which allowed factors secreted by ESC to diffuse to Hepa1-6 cells, without any direct contact.

Hepa1-6 cells $\left(1 \times 10^{6}\right)$ were co-cultured with ESC for $144 \mathrm{~h}$ and MTT assays were performed every $24 \mathrm{~h}$. By $72 \mathrm{~h}$, compared with MEFs (control group), ESCs caused inhibition of Hepa1-6 cell proliferation, and the effect was also observed at 96,120 and $144 \mathrm{~h}$ (Fig. 1A). To determine the effect of ESC microenvironment on Hepa1-6 cell proliferation, day 1 2 and 3 ESC-CM treatment and MEF-CM treatment were used for the MMT assay. The media in which Hepa1-6 cells were seeded into 96 -well plates and incubated for $24 \mathrm{~h}$ was replaced by day 12 or 3 ESC-CM or MEF-CM. MTT assays were performed every $24 \mathrm{~h}$. Day 1 ESC-CM suppressed Hepa1- 6 cell proliferation only at 120 and 144 h; however day 2 ESC-CM inhibited cell proliferation at 72, 96, 120
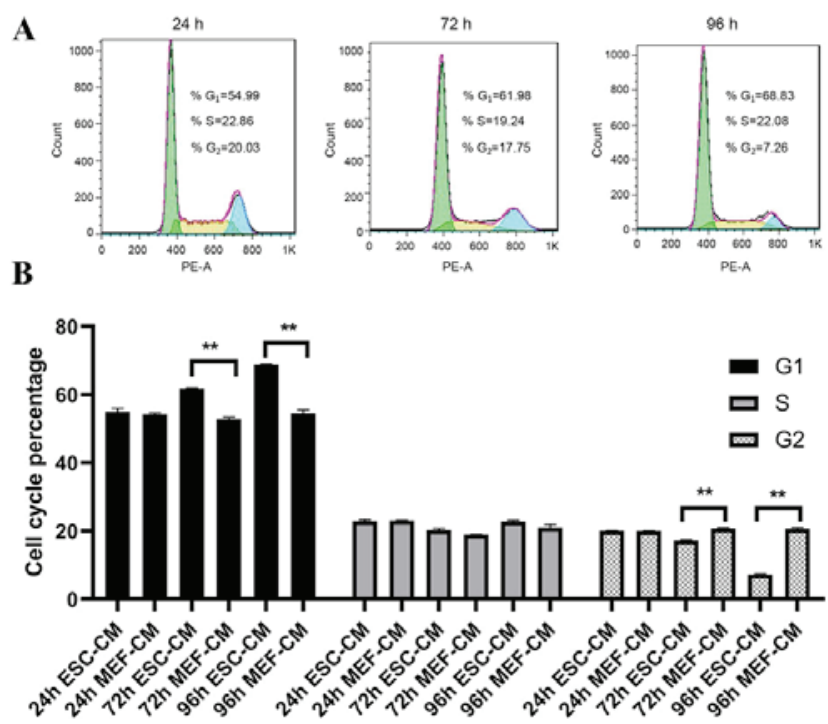

Figure 2.Effects of ESC-CM on cell cycle of Hepa1-6 cells. (A) Representative dot plots, showing cell cycle analysis of Hepa1-6 following 48-h ESC-CM treatment for 24, 72 and $96 \mathrm{~h}$ by flow cytometry. (B) Quantified data of (A). ESC-CM, embryonic stem cell-conditioned medium. ${ }^{* *} \mathrm{P}<0.01$. Green represents cell in the $G_{1}$ phase; Yellow represents cell in $S$ phase and blue represents cells in the $\mathrm{G}_{2}$ phase.

and $144 \mathrm{~h}$ (Fig. 1B and D). Furthermore, the day 3 ESC-CM resulted in stronger inhibition than day 2 ESC-CM, with a significant inhibition of cell proliferation at 48, 72, 96, 120 and $144 \mathrm{~h}$ (Fig. 1C). 

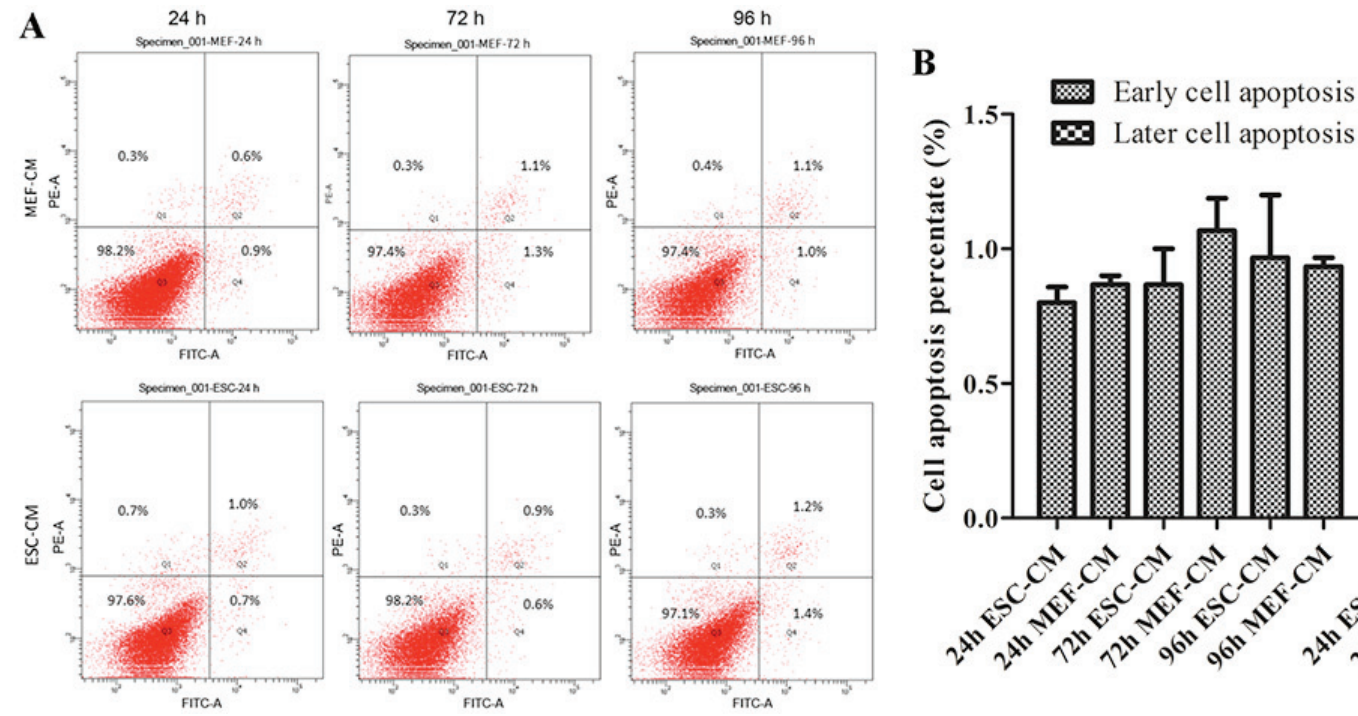

Figure 3. ESC-CM does not induce apoptosis. (A) Representative dot plots of Hepa1-6 cells were treated with 48-h ESC-CM or MEF-CM for 24, 72 and 96 h. The cells were harvested and processed for apoptosis assay using an annexin V-FITC apoptosis detection kit. The percentages of late apoptotic and early apoptotic cells are displayed in Q2 and Q4. (B) Quantified data of (A) ESC-CM, embryonic stem cell-conditioned medium; FITC, fluorescein isothiocyanate; MEF-CM, mouse embryonic fibroblast-conditioned medium.

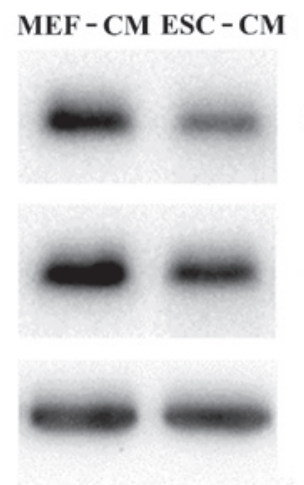

\section{Cyclin D1}

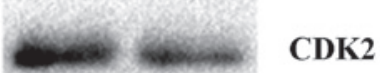

Cyclin E1

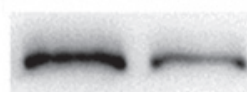

CDK4

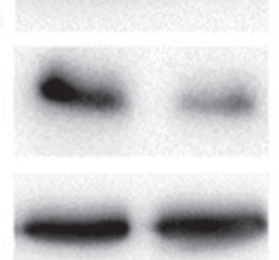

CDK6

$\beta$-actin

\section{$\beta$-actin}

Figure 4. ESC-CM downregulates $\mathrm{G}_{1}$ phase cell cycle-associated protein. Hepa1-6 cells were treated with 72-h ESC-CM and MEF-CM for 72 h, followed by western blotting with indicated antibodies. $\beta$-actin served as a loading control. ESC-CM, embryonic stem cell conditioned medium; MEF-CM, mouse embryonic fibroblast conditioned medium.

ESC-CM arrests cells at $G_{1}$ phase of the cell cycle. In order to understand how ESC-CM inhibits Hepa1-6 cell proliferation, cell cycle analysis was performed. Cells were treated with day 2 ESC-CM or MEF-CM (control group) and harvested following 24-96 $\mathrm{h}$ and stained with PI for fluorescence-activated cell sorting analysis. The results indicated an increased Hepa1-6 cell number in $\mathrm{G}_{1}$ phase from $54.99 \pm 0.95$ (24 h ESC-CM) to $68.83 \pm 0.18 \%$ (96 $\mathrm{h}$ ESC-CM) $(\mathrm{P}<0.001)$ and a reduction in cell numbers in $\mathrm{G}_{2} / \mathrm{M}$ phase from $20.03 \pm 0.38 \%$ (24 h ESC-CM) to $7.26 \pm 0.16 \%$ (96 h ESC-CM) ( $<<0.001$; Fig. 2). These findings indicate that ESC-CM inhibited cell proliferation by arresting cells at $\mathrm{G}_{1}$ phase of the cell cycle.

Effect of ESC-CM on cell apoptosis. In order to further investigate whether ESC-CM could inhibit cell proliferation through increased apoptosis, Hepa1-6 cells were treated with day 2 ESC-CM or MEF-CM for 24-96 h prior to harvesting. The cells were stained with Annexin V-FITC and PI and were analyzed by flow cytometry. There were no significant differences in the number of early apoptotic cells $(1.0 \pm 0.09 \%)$ at $24 \mathrm{~h}$ and in the number of late apoptotic cells $(1.2 \pm 0.03 \%)$ at $96 \mathrm{~h}$ in cells treated with ESC-CM compared with MEF-CM (early apoptotic cells, $0.6 \pm 0.07 \%$; late apoptotic cells, $1.1 \pm 0.03 \%$ ) at the end of the experiment. These data indicate that the ESC-CM did not mediate its growth inhibitory effects through increased apoptosis (Fig. 3).

ESC-CM downregulates the expression of $G_{I}$ phase-associated $C D K s$. The mechanism of arresting cells at the $\mathrm{G}_{1}$ phase by ESC-CM was investigated by determining the expression of CDKs, including Cyclin D1, Cyclin E1, CDK2, CDK4 and CDK6. As shown in Fig. 4, treatment with day 2 ESC-CM for $72 \mathrm{~h}$ resulted in downregulated cellular protein expression of Cyclin D1, CDK2, Cyclin E1, CDK4 and CDK6 compared with Hepa1-6 cells in MEF-CM for $72 \mathrm{~h}$. These findings suggest that ESC-CM arrested Hepa1- 6 cells in the $\mathrm{G}_{1}$ phase of the cell 

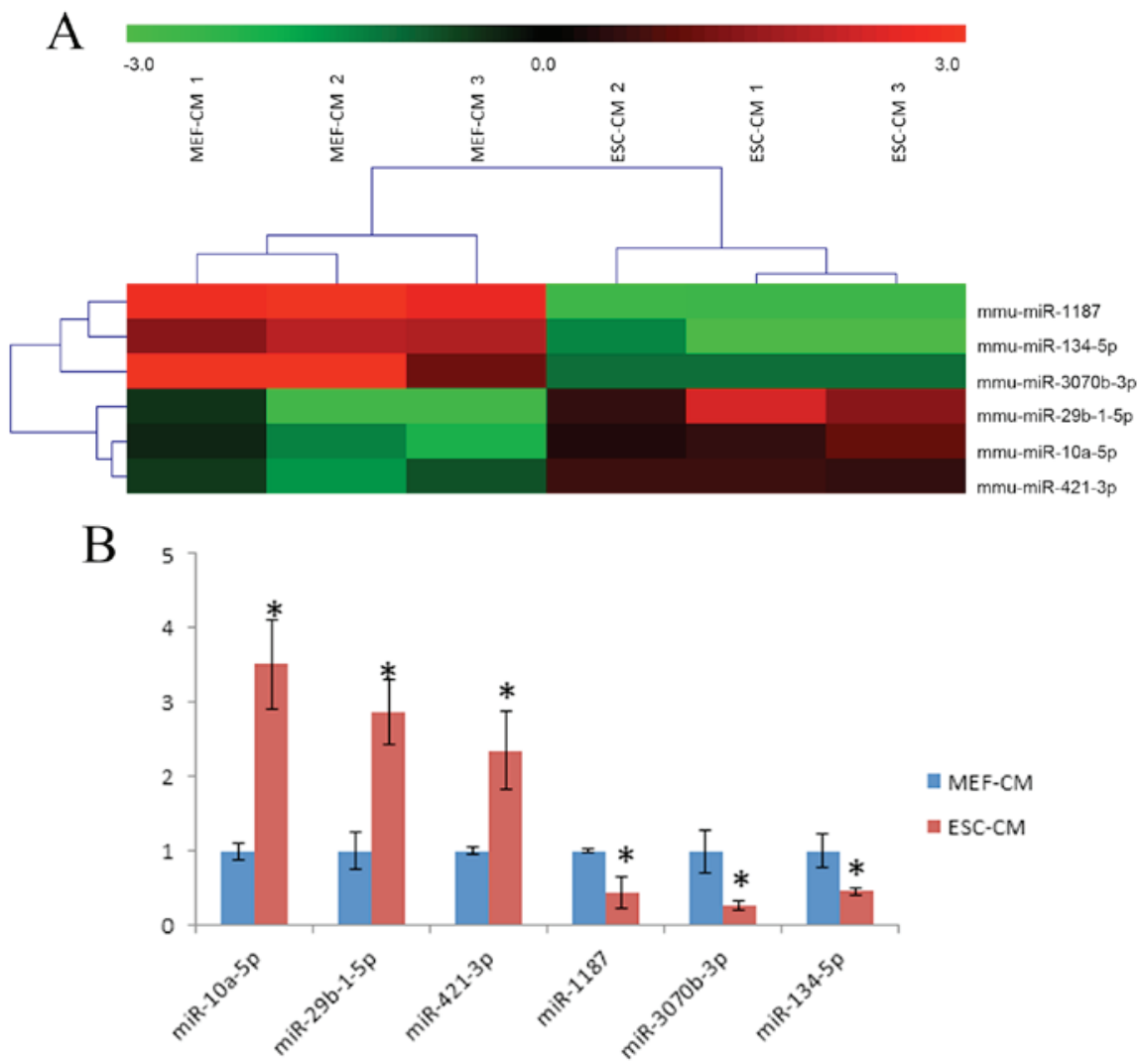

Figure 5. Differential expression of miRNAs between Hepa1-6 cells treated with embryonic stem cell-conditioned medium and control. (A) Heatmap of differentially expressed miRNA according to the miRNA array results (red represents high expression; green represent low expression). (B) Relative expression levels of the differentially expressed miRNA determined by reverse transcription-quantitative PCR. "P $<0.05$. miRNA, microRNA; ESC-CM, embryonic stem cell conditioned medium; MEF-CM, mouse embryonic fibroblast conditioned medium.

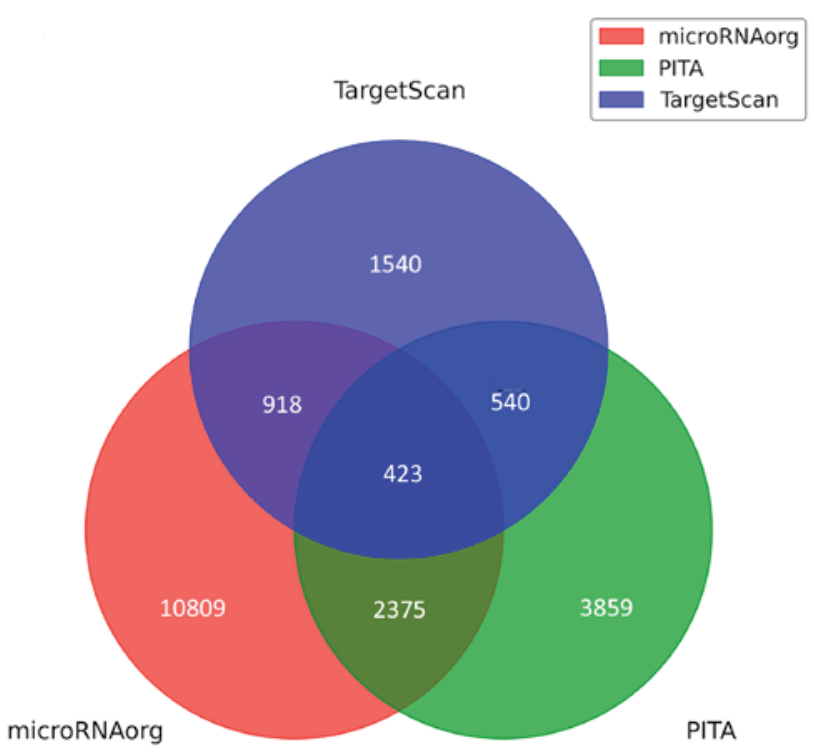

Figure 6. Venn diagram of predicted target gene numbers by three databases. In total, 423 common target genes were predicted by TargetScan, PITA and microRNAorg, according to the differentially expressed miRNAs.

cycle by downregulating the expression of $\mathrm{G}_{1}$ phase-associated cyclin D1 and CDKs.

miRNA profile. In order to further explore the effect of ESC-CM on miRNA expression in Hepa1-6 cells, the miRNA profile of Hepal-6 cells cultured with 72-h ESC-CM were compared with the control group co-cultured with 72-h MEF-CM. A total of 6 differentially expressed miRNAs were found in the ESC-CM group, specifically three miRNAs (miR-29b-1-5p, miR-10a-5p and miR-421-3p) were upregulated and three miRNAs (miR-1187, miR-134-5p and miR-3070b-3p) were downregulated (Fig. 5A). These results were subsequently confirmed by RT-qPCR analysis (Fig. 5B).

A total of 423 predicted target genes were obtained according to the differentially expressed miRNAs (Fig. 6). These target genes were particularly enriched in the 'cell cycle' (CCND2, CDK4, CDK14 and Notch2), 'apoptotic process' and 'signal transduction' (Fig. 7). The results of the KEGG analysis revealed the 'Wnt signaling pathway' and the 'Hippo signaling pathway' to be particularly enriched (Fig. 8).

\section{Discussion}

To date, the mechanism of tumor occurrence, development, metastasis and recurrence is not completely elucidated, which is a complex biological process and associated with genomic instability, chromosomal abnormalities and genetic mutations. With the in-depth study of tumors, it was found that the occurrence, development and metastasis were associated with the surrounding environment of tumors (13). There is a biphasic and dynamic interaction between the tumor microenvironment and tumor cells (15). Studies have shown that the embryonic microenvironment can alter the biological behavior 


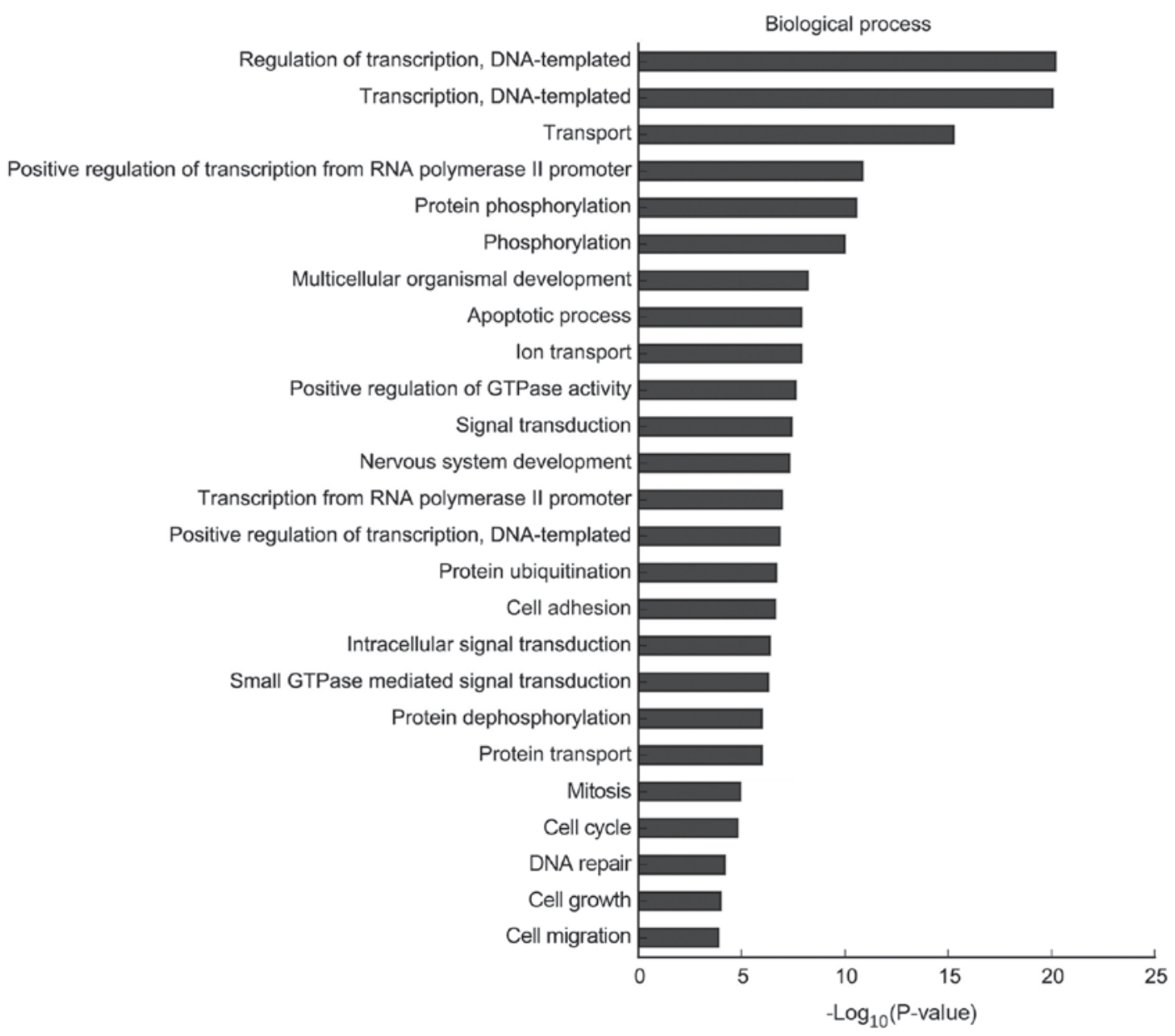

Figure 7. Enrichment of predicted target genes in the GO database. The predicted target genes were enriched in the 'cell cycle' (CCND2, CDK4, CDK14 and Notch2), 'apoptotic process' and 'signal transduction' by GO analysis (top 20 shown). GO, gene ontology.

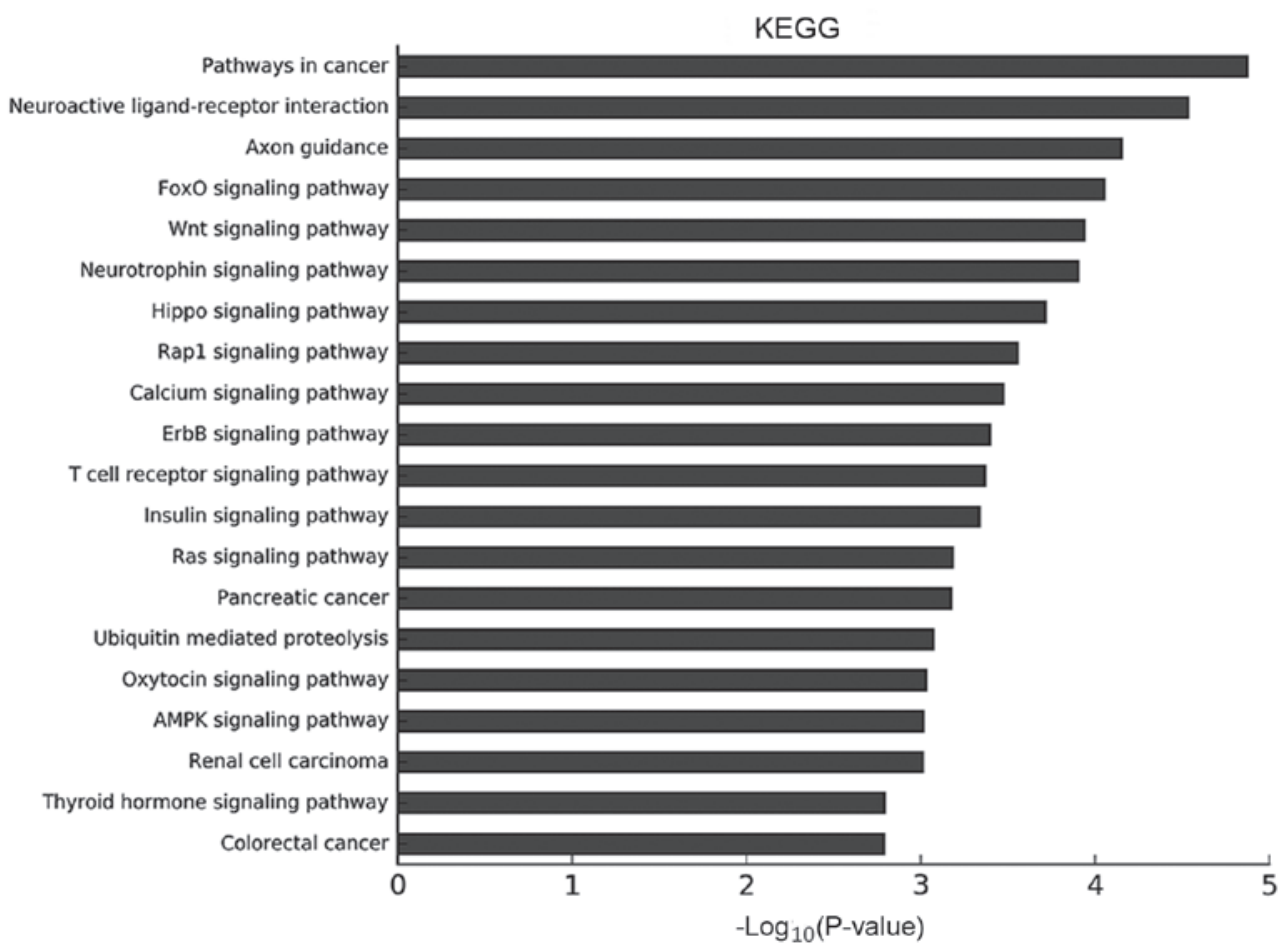

Figure 8. Enrichment of predicted target genes in the KEGG database. Top 20 results from the KEGG analysis. KEGG, Kyoto Encyclopedia of Genes and Genomes. 
of malignant tumor cells, and that cytokines secreted by ESCs regulate the proliferation and invasion of some tumor cells $(15,16)$. In addition, owing to fewer ESC antigens being expressed in normal tissues compared with traditional cancer vaccines, ESCs cause fewer autoimmune responses while killing tumor cells (17).

In the present study, 24-h CM from ESCs was demonstrated to inhibit the proliferation of hepatocellular carcinoma cells after $120 \mathrm{~h}$, whereas 72-h CM inhibited the proliferation of hepatocellular carcinoma cells at $48 \mathrm{~h}$. All CM inhibited the proliferation of HCC cells, but the inhibitory ability of $\mathrm{CM}$ at different co-culture durations was different, which may be associated with the concentration of bioactive substances secreted by ESCs. Furthermore, ESC-CM blocked hepatocellular carcinoma cells at the $G_{1}$ phase, whereas no significant differences were observed in the apoptosis of Hepa1-6 cells. Furthermore, the expression levels of $\mathrm{G}_{1}$-associated regulatory proteins (cyclin D1/CDK4/CDK6) in the ESC-CM group were significantly lower compared with that in the MEF-CM group.

In order to explore the mechanism further, six significantly differentially expressed miRNAs were identified in the group with 48-h ESC-CM treatment compared with the group with 72-h MEF-CM treatment. Furthermore, 423 putative target genes of the regulated miRNAs were predicted. Among them, miR-134-5p has been reported to function as a tumor suppressor gene in gastric cancer (18), and inhibit proliferation and promote apoptosis of lung cancer cells by inhibiting the ERK1/2 signaling pathway (19). High expression of miR-29b-1-5p was demonstrated to inhibit tumor cell proliferation and migration in breast cancer (20). miR-1187 may play a role in viral hepatitis (21). In a previous study, miR-421-3p was abnormally expressed in post-traumatic stress disorder (22). miR-10a-5p can function as an oncogene or a tumor suppressor gene depending on the cell type, which demonstrated that miR-10a-5p had tumor-specific and time-space specificities (16-18). To the best of our knowledge the biological function of miR-3070b-3p was not reported in the previous literature.

The KEGG analysis revealed the importance of the Wnt signaling pathway in the occurrence and development of liver cancer. In a previous study, Wnt/ $\beta$-catenin signaling pathway was an important factor in the early development and progression of liver cancer (23). Furthermore, some studies have shown that non-canonical Wnt signaling pathway can inhibit the proliferation and metastasis of hepatoma cells by antagonizing the classical Wnt signaling pathway (24,25). CDK14 was highly expressed in hepatocarcinoma tissues, which was associated with the invasion function of hepatoma cells (26). CDK14 can regulate cell cycle and cell proliferation by interacting with cyclin D3 and cyclin Y (27,28). The complexes of CDK14 and cyclin Y can activate the non-canonical Wnt signaling pathway in liver cancer cells (29). In addition, the Hippo signaling pathway also plays an important role in cell proliferation, apoptosis, cell cycle and differentiation (30). The screening for effective miRNAs that regulate the Wnt signaling pathway may be a new approach for liver cancer treatment.

In conclusion, ESC-CM was demonstrated to regulate the expression of cyclin D1/CDK4/CDK6 and the Wnt and Hippo signaling pathways through miRNAs, and resulted in cell cycle arrest at the $G_{1}$ phase and inhibited cell proliferation of Hepa1-6 cells.

\section{Acknowledgements}

Not applicable.

\section{Funding}

No funding was received.

\section{Availability of data and materials}

The datasets used and/or analyzed during the present study are available from the author on reasonable request.

\section{Authors' contributions}

LL and YZ designed the study and carried out the experiments. LL, YZ and QZ performed the analysis. JJ participated in the design of the study. All authors read and approved the final version of the manuscript.

\section{Ethical approval and consent to participate}

Not applicable.

\section{Patient consent for publication}

Not applicable.

\section{Competing interests}

The authors declare that they have no competing interests.

\section{References}

1. Bray F, Ferlay J, Soerjomataram I, Siegel RL, Torre LA and Jemal A: Global cancer statistics 2018: GLOBOCAN estimates of incidence and mortality worldwide for 36 cancers in 185 countries. CA Cancer J Clin 68: 394-424, 2018.

2. Ryan MJ, Willatt J, Majdalany BS, Kielar AZ, Chong S, Ruma JA and Pandya A: Ablation techniques for primary and metastatic liver tumors. World J Hepatol 8: 191-199, 2016.

3. Boroviak T, Loos R, Bertone P, Smith A and Nichols J: The ability of inner-cell-mass cells to self-renew as embryonic stem cells is acquired following epiblast specification. Nat Cell Biol 16: 516-528, 2014.

4. Williams JW III, Carlson DL, Gadson RG, Rollins-Smith L, Williams CS and Mckinnell RG: Cytogenetic analysis of triploid renal carcinoma in Rana pipiens. Cytogenet Cell Genet 64: 18-22, 1993.

5. Illmensee K: Reversion of malignancy and normalized differentiation of teratocarcinoma cells in chimeric mice. Basic Life Sci 12: 3-25, 1978

6. Melzer C, von der Ohe J, Lehnert H, Ungefroren $\mathrm{H}$ and Hass R: Cancer stem cell niche models and contribution by mesenchymal stroma/stem cells. Mol Cancer 16: 28, 2017.

7. Giuffrida D, Rogers IM, Nagy A, Calogero AE, Brown TJ and Casper RF: Human embryonic stem cells secrete soluble factors that inhibit cancer cell growth. Cell Prolif 42: 788-798, 2009.

8. Cavallari C, Fonsato V, Herrera MB, Bruno S, Tetta C and Camussi G: Role of lefty in the anti tumor activity of human adult liver stem cells. Oncogene 32: 819-826, 2013.

9. He N, Feng G, Li Y, Xu Y, Xie X, Wang H, Wang Y, Ou L, Pei X and Liu N: Embryonic stem cell preconditioned microenvironment suppresses tumorigenic properties in breast cancer. Stem Cell Res Ther 7: 95, 2016

10. Becker A, Thakur BK, Weiss JM, Kim HS, Peinado H and Lyden D: Extracellular vesicles in cancer: Cell-to-cell mediators of metastasis. Cancer Cell 30: 836-848, 2016. 
11. Gopal SK, Greening DW, Rai A, Chen M, Xu R, Shafiq A, Mathias RA, Zhu HJ and Simpson RJ: Extracellular vesicles: Their role in cancer biology and epithelial-mesenchymal transition. Biochem J 474: 21-45, 2017.

12. Khan M, Nickoloff E, Abramova T, Johnson J, Verma SK, Krishnamurthy P, Mackie AR, Vaughan E, Garikipati VN, Benedict C, et al: Embryonic stem cell-derived exosomes promote endogenous repair mechanisms and enhance cardiac function following myocardial infarction. Circ Res 117: 52-64, 2015.

13. Yaddanapudi K, Mitchell RA, Putty K, Willer S, Sharma RK, Yan J, Bodduluri H and Eaton JW: Vaccination with embryonic stem cells protects against lung cancer: Is a broad-spectrum prophylactic vaccine against cancer possible? PLoS One 7: e42289, 2012.

14. Livak KJ and Schmittgen TD: Analysis of relative gene expression data using real-time quantitative PCR and the 2(-Delta Delta C(T)) method. Methods 25: 402-408, 2001.

15. Zhang ZJ, Chen XH, Chang XH, Ye X, Li Y and Cui H: Human embryonic stem cells-a potential vaccine for ovarian cancer. Asian Pac J Cancer Prev 13: 4295-4300, 2012.

16. Zhang Z, Chen X, Chang X, Ye X, Li Y and Cui H: Vaccination with embryonic stem cells generates effective antitumor immunity against ovarian cancer. Int J Mol Med 31: 147-153, 2013.

17. Adam V, Wauters I and Vansteenkiste J: Melanoma-associated antigen-A3 vaccination in the treatment of non-small-cell lung cancer. Exp Opin Biol Ther 14: 365-376, 2014.

18. Qiu ZA and He GP: MicroRNA-134 functions as a tumor suppressor gene in gastric cancer. Am J Transl Res 8: 4320-4328, 2016.

19. Chen T, Gao F, Feng S, Yang T and Chen M: MicroRNA-134 regulates lung cancer cell $\mathrm{H} 69$ growth and apoptosis by targeting WWOX gene and suppressing the ERK1/2 signaling pathway. Biochem Biophys Res Commun 464: 748-754, 2015.

20. Muluhngwi P, Krishna A, Vittitow SL, Napier JT, Richardson KM, Ellis M, Mott JL and Klinge CM: Tamoxifen differentially regulates miR-29b-1 and miR-29a expression depending on endocrine-sensitivity in breast cancer cells. Cancer Lett 388: 230-238, 2017.
21. Yu DS, An FM, Gong BD, Xiang XG, Lin LY, Wang H and Xie Q: The regulatory role of microRNA-1187 in TNF- $\alpha$-mediated hepatocyte apoptosis in acute liver failure. Int J Mol Med 29: 663-668, 2012.

22. Balakathiresan NS, Chandran R, Bhomia M, Jia M, Li H and Maheshwari RK: Serum and amygdala microRNA signatures of posttraumatic stress: Fear correlation and biomarker potential. J Psychiatr Res 57: 65-73, 2014.

23. Liu LJ, Xie SX, Chen YT, Xue JL, Zhang CJ and Zhu F: Aberrant regulation of Wnt signaling in hepatocellular carcinoma. World J Gastroenterol 22: 7486-7499, 2016.

24. Yuzugullu H, Benhaj K, Ozturk N, Senturk S, Celik E, Toylu A, Tasdemir N, Yilmaz M, Erdal E, Akcali KC, et al: Canonical Wnt signaling is antagonized by noncanonical Wnt5a in hepatocellular carcinoma cells. Mol Cancer 8: 90, 2009.

25. Toyama T, Lee HC, Koga H, Wands JR and Kim M: Noncanonical Wnt11 inhibits hepatocellular carcinoma cell proliferation and migration. Mol Cancer Res 8: 254-265, 2010.

26. Pang EY, Bai AH, To KF, Sy SM, Wong NL, Lai PB, Squire JA and Wong N: Identification of PFTAIRE protein kinase 1, a novel cell division cycle-2 related gene, in the motile phenotype of hepatocellular carcinoma cells. Hepatology 46: 436-445, 2010.

27. Shu F, Lv S, Qin Y, Ma X, Wang X, Peng X, Luo Y, Xu BE, Sun $\mathrm{X}$ and $\mathrm{Wu}$ J: Functional characterization of human PFTK1 as a cyclin-dependent kinase. Proc Natl Acad Sci USA 104: 9248-9253, 2007.

28. Jiang M, Gao Y, Yang T, Zhu X and Chen J: Cyclin Y, a novel membrane-associated cyclin, interacts with PFTK1. FEBS Lett 583: 2171-2178, 2009

29. Sun T, Co NN and Wong N: PFTK1 interacts with cyclin $Y$ to activate non-canonical Wnt signaling in hepatocellular carcinoma. Biochem Biophys Res Commun 449: 163-168, 2014.

30. Wang H, Du YC, Zhou XJ, Liu H and Tang SC: The dual functions of YAP-1 to promote and inhibit cell growth in human malignancy. Cancer Metastasis Rev 33: 173-181, 2014.

This work is licensed under a Creative Commons Attribution-NonCommercial-NoDerivatives 4.0 International (CC BY-NC-ND 4.0) License. 